Proyecciones Journal of Mathematics

Vol. 32, No 1, pp. 15-29, March 2013.

Universidad Católica del Norte

Antofagasta - Chile

\title{
Generalized Ulam-Hyers-Rassias stability of a Cauchy type functional equation
}

\author{
Mohamed Akkouchi \\ Cadi Ayyad University, Maroc \\ Received : November 2012. Accepted : December 2012
}

\begin{abstract}
Using the alternative fixed point theorem, we establish the generalized Hyers-Ulam-Rassias stability of a Cauchy type functional equation for functions taking values in arbitrary complete (real or complex) $\beta$-normed spaces.
\end{abstract}

Subjclass [2000] : 39B10, 26D20, 39B70, 47H10.

Keywords : Alternative fixed point, Generalized Hyers-Ulam-Rassias stability, Cauchy type functional equation, additive mappings, $\beta$-normed spaces. 


\section{Introduction}

In 1940, S. M. Ulam (see [30]) proposed the following problem:

Given a group $G_{1}$, a metric group $\left(G_{2}, d\right)$ and a positive number $\epsilon$, does there exist a $\delta>0$ such that if a function $f: G_{1} \longrightarrow G_{2}$ satisfies the inequality $d(f(x y), f(x) f(y))<\delta$ for all $x, y \in G_{1}$, then there exists a homomorphism $T: G_{1} \rightarrow G_{2}$ such that $d(f(x), T(x))<\epsilon$ for all $x \in G_{1}$ ?

When this problem has a solution, we say that the homomorphisms from $G_{1}$ to $G_{2}$ are stable or that the functional equation defining homomorphisms is stable in the sense of Ulam.

In 1941, D. H. Hyers [14] gave a partial solution of Ulam's problem for the case of approximate additive mappings under the assumption that $G_{1}$ and $G_{2}$ are Banach spaces. Indeed, he proved that each solution of the inequality

$$
\|f(x+y)-f(x)-f(x)\| \leq \epsilon, \quad \forall x, y \in G_{1}
$$

can be approximated by an exact solution. That is by an additive mapping.

In 1950, T. Aoki [2] was the second author to study this problem for additive mappings.

In 1978, Th. M. Rassias [21] generalized the result of Hyers by considering the stability problem for unbounded Cauchy differences. This phenomenon of stability introduced by Th. M. Rassias [21] is called the Hyers-Ulam-Rassias stability.

Theorem 1.1. (Th. M. Rassias [21]) Let $f: E_{1} \longrightarrow E_{2}$ be a mapping from a real normed vector space $E_{1}$ into a Banach space $E_{2}$ satisfying the inequality

$$
\|f(x+y)-f(x)-f(y)\| \leq \epsilon\left(\|x\|^{p}+\|y\|^{p}\right)
$$

for all $x, y \in E_{1}$, where $\epsilon$ and $p$ are constants with $\epsilon>0$ and $p<1$. Then there exists a unique additive mapping $T: E_{1} \longrightarrow E_{2}$ such that

$$
\|f(x)-T(x)\| \leq \frac{2 \epsilon}{2-2^{p}}\|x\|^{p}, \quad \forall x \in E_{1} .
$$

If $p<0$ then inequality (1.1) holds for all $x, y \neq 0$, and (1.2) for $x \neq 0$. Also, if the function $t \mapsto f(t x)$ from $R$ into $E_{2}$ is continuous for each fixed $x \in E$, then $T$ is linear.

In [13], Gajda considered also the stability problem with unbounded Cauchy differences. From the papers of Hyers, Rassias and Gajda, we have the following Theorem which completes the results of Theorem 1.1. 
Theorem 1.2. (Hyers-Rassias-Gajda) [[13], [15], [21]].

Suppose that $E_{1}$ is a real normed space, $E_{2}$ is a real Banach space, $f: E_{1} \longrightarrow E_{2}$ is a given function, and the following condition holds

$$
\|f(x+y)-f(x)-f(y)\|_{E_{2}} \leq \theta\left(\|x\|_{E_{1}}^{p}+\|y\|_{E_{1}}^{p}\right), \quad \forall x, y \in E_{1}, \quad\left(C_{p}\right)
$$

for some $p \in[0,+\infty) \backslash\{1\}$.

Then there exists a unique additive function $T: E_{1} \longrightarrow E_{2}$ such that

$$
\|f(x)-T(x)\|_{E_{2}} \leq \frac{2 \theta}{\left|2-2^{p}\right|}\|x\|_{E_{1}}^{p}, \quad \forall x \in E_{1} . \quad\left(\text { Est }_{p}\right)
$$

It is worth noting that many of the subsequent proofs for Hyers-UlamRassias stability used the Hyers method. Namely, the function $T: E_{1} \longrightarrow$ $E_{2}$ is explicitly constructed, starting from the given function $f$, by the formulae

$$
T(x)=\lim _{n \rightarrow \infty} \frac{1}{2^{n}} f\left(2^{n} x\right), \quad \text { if } \quad p<1,
$$

and

$$
T(x)=\lim _{n \rightarrow \infty} 2^{n} f\left(\frac{x}{2^{n}}\right), \quad \text { if } \quad p>1 .
$$

This method is called a direct method.

We observe that the estimate $\left(E s t_{p}\right)$ depends on the parameter $p$.

There exist also other approaches, see for example the invariant mean technique introduced by Szekelyhidi (see e.g. [[27], [28]]), or based on the sandwich theorems (see [18]). The interested reader is invited to consult the expository papers [[12], [23], [29]] and the book [15].

In [20], V. Radu obtained some stability results via the alternative fixed point theorem. In their paper [6], L. Cădariu and V. Radu have used the same fixed point method to establish the stability of functional equations of Jensen type.

It is worth noting that J. A. Baker [3] has started the use of fixed point theorems in the stability theory. In [3], Baker used the Banach principle to study Ulam-Hyers stability of a class of nonlinear functional equations. The results of [3] were extended by M. Akkouchi (see [1]) by using a Cirić fixed point theorem.

Fixed point methods were applied with success to various functional equations. See for example [8], [5], [17], [19], [20] and others.

The aim of this paper is to apply the alternative fixed point theorem to deal with functional equations of Cauchy type. That is starting from 
initial conditions of the form

$(C(k, \varphi)) \quad\left\|\frac{1}{k} f(k(x+y))-f(x)-f(y)\right\|_{F} \leq \varphi(x, y), \quad \forall x, y \in G$,

where $k$ is a given positive integer, $F$ is a $\beta$-complete normed space over the real or complex field $\mathbf{K}, G$ is a linear space over $\mathbf{K}$ and $\varphi$ is a control function.

In particular, our results extend both Theorem 1.1 and Theorem 1.2 to the case of functions taking values in arbitrary complete (real or complex) $\beta$-normed linear spaces.

\section{Preliminaries}

For a nonempty set $X$, we recall the definition of the generalized metric on $X$. A function $d: X \times X \rightarrow[0, \infty]$ is called a generalized metric on $X$ if and only if $d$ satisfies

(M1) $d(x, y)=0$ if and only if $x=y$,

(M2) $d(x, y)=d(y, x)$ for all $x, y \in X$,

(M3) $d(x, z) \leq d(x, y)+d(y, z)$ for all $x, y, z \in X$.

We observe that the only one difference of the generalized metric from the usual metric is that the range of the former is allowed to include the infinity.

We now recall one of fundamental results of fixed point theory. For the proof, we refer to [11].

Theorem 2.1. (The alternative of fixed point [11])

Suppose we are given a complete generalized metric space $(X, d)$ and a strictly contractive mapping $\Lambda: X \rightarrow X$, with the Lipschitz constant $L$.

Then, for each given point $x \in X$, either

$\left(A_{1}\right): d\left(\Lambda^{n} x, \Lambda^{n+1} x\right)=+\infty, \quad \forall n \geq 0$,

or

$\left(A_{2}\right)$ : there exists a nonnegative integer $n_{0}$ such that:

(i) $d\left(\Lambda^{n} x, \Lambda^{n+1} x\right)<+\infty$ for all natural number $n \geq n_{0}$.

(ii) The sequence $\left\{\Lambda^{n} x\right\}$ converges to a fixed point $y_{*}$ of $\Lambda$.

(iii) $y_{*}$ is the unique fixed point of $\Lambda$ in the set

$$
X\left(x, n_{0}\right)=\left\{y \in X: d\left(\Lambda^{n_{0}} x, y\right)<\infty\right\} .
$$


(iv) If $y \in Y\left(x, n_{0}\right)$, then

$$
d\left(y, y_{*}\right) \leq \frac{1}{1-L} d(\Lambda y, y)
$$

Throughout this paper, we fix a real number $\beta$ with $0<\beta \leq 1$ and let $\mathbf{K}$ denote either $\mathbf{R}$ or $\mathbf{C}$. Suppose $F$ is a vector space over $\mathbf{K}$. A function $\|.\|_{\beta}: F \longrightarrow[0,+\infty)$ is called a $\beta$-norm if and only if it satisfies

(N1) $\|x\|_{\beta}=0$ if and only if $x=0$;

(N2) $\|\lambda x\|_{\beta}=|\lambda|^{\beta}\|x\|_{\beta}$ for all $\lambda \in \mathbf{K}$ and all $x \in F$;

(N3) $\|x+y\|_{\beta} \leq\|x\|_{\beta}+\|y\|_{\beta}$ for all $x, y \in F$.

\section{Main results}

Let $(G,+)$ be an abelian group. Let $k \geq 1$ be an integer. Let $F$ be a vector space over the (real or complex) field $\mathbf{K}$ endowed with a $\beta$-norm $\|\cdot\|_{\beta}$. For any function $f: G \longrightarrow F$, we consider the difference

$$
D_{k} f(x, y)=\frac{f(k(x+y))}{k}-f(x)-f(y),
$$

defined for all $x, y \in G$

In connection with this difference, we have the following Cauchy type functional equation:

$$
f(k(x+y))-k f(x)-k f(y)=0, \quad \forall x, y \in G .
$$

It is easy to prove the following lemma concerning the solutions of the equation (3.2).

Lemma 3.1. Let $(G,+)$ be an abelian group. Let $k \geq 1$ be an integer. Let $F$ be a vector space over the (real or complex) field $\mathbf{K}$ endowed with a $\beta$-norm $\|.\|_{\beta}$. Let a function $f: G \longrightarrow F$ be given.

Then the following assertions are equivalent:

(a) $f$ is additive. That is $f(x+y)=f(x)+f(y)$, for all $x, y \in G$.

(b) $f$ is a solution of the equation (3.2). That is $f(k(x+y))=k f(x)+$ $k f(y)$, for all $x, y \in G$.

The first main result of this paper reads as follows.

Theorem 3.2. Let $(G,+)$ be an abelian group. Let $k \geq 1$ be an integer. Let $\left(F,\|\cdot\|_{\beta}\right)$ be a complete $\beta$-normed vector space over the (real or complex) field $\mathbf{K}$ endowed with a $\beta$-norm $\|.\|_{\beta}$, where $0<\beta \leq 1$. Let $f: G \longrightarrow$ 
$F$ be a mapping for which there exists a function $\varphi: G \times G \rightarrow[0, \infty)$ such that

$$
\left\|D_{k} f(x, y)\right\|_{\beta} \leq \varphi(x, y), \quad \forall x, y \in G .
$$

We suppose also that there exists a constant $L, 0<L<1$ such that

$$
\varphi(2 k x, 2 k x) \leq(2 k)^{\beta} L \varphi(x, x), \quad \forall x \in G
$$

and

$$
\lim _{n \rightarrow \infty} \frac{\varphi\left(2^{n} k^{n} x, 2^{n} k^{n} y\right)}{(2 k)^{n \beta}}=0, \quad \forall x, y \in G .
$$

Then there exists a unique additive mapping $f^{*}: G \rightarrow F$ such that

$$
\left\|f(x)-f^{*}(x)\right\|_{\beta} \leq \frac{1}{2^{\beta}} \frac{1}{1-L} \varphi(x, x)
$$

for all $x \in G$.

Proof. We consider the set

$$
\mathbf{X}=\{h: G \rightarrow F\}
$$

For each pair $\{g, h\}$ of elements of $\mathbf{X}$, we define

$$
d_{\varphi}(g, h):=\inf \left\{c \in[0, \infty):\|g(x)-h(x)\|_{\beta} \leq c \varphi(x, x), \text { for all } x \in G\right\},
$$

with the convention $\inf \emptyset:=+\infty$. Then it is easy to see that $d_{\varphi}$ is a generalized distance on the set $\mathbf{X}$. As in [7], one can prove that the generalized metric space $\left(\mathbf{X}, d_{\varphi}\right)$ is complete.

We define an operator $\Lambda: \mathbf{X} \rightarrow \mathbf{X}$ by

$$
(\Lambda h)(x)=\frac{1}{2 k} h(2 k x),
$$

for all $x \in G$.

First, we start by proving that $\Lambda$ is strictly contractive on the $\left(\mathbf{X}, d_{\varphi}\right)$. To this end, let $g, h \in \mathbf{X}$ be given. Without loss of generality, we may suppose that $d_{\varphi}(g, h)$ is finite. In this case, let $c \in[0, \infty)$ be any arbitrary constant such that

$$
\|g(x)-h(x)\|_{\beta} \leq c \varphi(x, x), \quad \forall x \in G .
$$


By replacing $x$ in the last inequality by $2 k x$ and making use of (3.4), we have

$\|(\Lambda g)(x)-(\Lambda h)(x)\|_{\beta}=\left\|\frac{g(2 k x)-h(2 k x)}{2 k}\right\|_{\beta} \leq c \frac{\varphi(2 k x, 2 k x)}{(2 k)^{\beta}} \leq \operatorname{Lc\varphi }(x, x)$,

for every $x \in G$, i.e, $d_{\varphi}(\Lambda g, \Lambda h) \leq L c$. This implies that

$$
d_{\varphi}(\Lambda g, \Lambda h) \leq L d_{\varphi}(g, h), \quad \forall g, h \in \mathbf{X} .
$$

Next, we prove that $d_{\varphi}(\Lambda f, f)$ is finite. To this end, we set $x=y$ in (3.3). We get

$$
2^{\beta}\left\|\frac{1}{2 k} f(2 k x)-f(x)\right\|_{\beta}=\left\|\frac{1}{k} f(2 k x)-2 f(x)\right\|_{\beta} \leq \varphi(x, x), \forall x \in G,
$$

which implies that

$$
d_{\varphi}(\Lambda f, f) \leq \frac{1}{2^{\beta}}<\infty .
$$

Thus, we may apply Theorem 2.1. It follows that there exists a unique function $f^{*}$ in the set $\mathbf{X}(f, 0)$ (see Theorem 2.1) which is fixed by $\Lambda$, i.e, $\Lambda\left(f^{*}\right)=f^{*}$ such that $\lim _{n \rightarrow \infty} d_{\varphi}\left(\Lambda^{n} g, f^{*}\right)=0$ for each $g \in \mathbf{X}(f, 0)$. In particular, since $f \in \mathbf{X}(f, 0)$, we have $\lim _{n \rightarrow \infty} d_{\varphi}\left(\Lambda^{n} f, f^{*}\right)=0$, from which we deduce that

$$
\lim _{n \rightarrow \infty} \frac{1}{2^{n} k^{n}} f\left(2^{n} k^{n} x\right)=f^{*}(x), \quad \forall x \in G .
$$

From (iv) of Theorem 2.1, we obtain

$$
d_{\varphi}\left(f, f^{*}\right) \leq \frac{1}{1-L} d_{\varphi}(\Lambda f, f) \leq \frac{1}{2^{\beta}} \frac{1}{1-L},
$$

which implies that the inequality (3.6) is true for all $x \in G$.

Now, we prove that $f^{*}$ is additive. To this respect, we start by substituting $2^{n} k^{n} x$ and $2^{n} k^{n} y$ for $x$ and $y$ in (3.3), respectively. We obtain

$$
\left\|\frac{1}{k} f\left(k\left(2^{n} k^{n} x+2^{n} k^{n} y\right)\right)-f\left(2^{n} k^{n} x\right)-f\left(2^{n} k^{n} y\right)\right\|_{\beta} \leq \varphi\left(2^{n} k^{n} x, 2^{n} k^{n} y\right),
$$

which gives after dividing by $(2 k)^{n \beta}$ the following inequality

$$
\left\|\frac{1}{k} \Lambda^{n}(f)(k(x+y))-\Lambda^{n}(f)(x)-\Lambda^{n}(f)(y)\right\|_{\beta} \leq \frac{\varphi\left(2^{n} k^{n} x, 2^{n} k^{n} y\right)}{(2 k)^{n \beta}},
$$


for all $x, y \in G$.

According to (3.5) and (3.8), by letting $n$ tend to infinity in (3.10), it follows that

$$
f^{*}(k(x+y))=k f^{*}(x)+k f^{*}(y), \quad \forall x, y \in G .
$$

Thus $f^{*}$ satisfies the functional equation (3.2). By Lemma 3.1, we know that $f^{*}$ must be additive.

Finally, we prove that $f^{*}$ is uniquely determined. Assume that inequality (3.6) is also satisfied with another additive function $f^{\sharp}: G \rightarrow F$ besides $f^{*}$. As $f^{\sharp}$ is an additive function, $f^{\sharp}$ satisfies that

$$
\left(\Lambda f^{\sharp}\right)(x)=\frac{1}{2 k} f^{\sharp}(2 k x)=f^{\sharp}(x), \quad \forall x \in G .
$$

That is, $f^{\sharp}$ is a fixed point of $\Lambda$. Since $f^{\sharp}$ satisfies (3.6), it follows that

$$
d_{\varphi}\left(f, f^{\sharp}\right) \leq \frac{1}{2^{\beta}} \frac{1}{1-L}<\infty .
$$

By using the triangle inequality, we have

$$
d_{\varphi}\left(\Lambda f, f^{\sharp}\right) \leq d_{\varphi}(\Lambda f, f)+d_{\varphi}\left(f, f^{\sharp}\right)<\infty .
$$

Hence $f^{\sharp}$ is another fixed point of $\Lambda$ which belongs to the set

$$
\mathbf{X}(f, 0)=\left\{g \in \mathbf{X}: d_{\varphi}(\Lambda f, g)<\infty\right\} .
$$

Thus, Theorem 3.3 (iv) implies that $f^{\sharp}=f^{*}$. This proves the uniqueness of $f^{*}$ and completes the proof.

When the control function $\phi$ satisfies the conditions (3.4) and (3.5), the solution $f^{*}$ to the problem of Ulam for the Cauchy type equation (3.2) is given by the formulae

$$
f^{*}(x)=\lim _{n \rightarrow \infty} \frac{1}{2^{n} k^{n}} f\left(2^{n} k^{n} x\right), \quad \forall x \in G .
$$

If we replace the conditions (3.4) and (3.5) by the conditions (3.12) and (3.13) given below, then we obtain a similar formulae. Precisely, we prove in the next theorem that under these conditions, the solution $f^{*}$ to the problem of Ulam for the equation (3.2) is given by the formulae

$$
f^{*}(x)=\lim _{n \rightarrow \infty} 2^{n} k^{n} f\left(\frac{x}{2^{n} k^{n}}\right), \quad \forall x \in G .
$$


Theorem 3.3. Let $G$ and $\left(F,\|\cdot\|_{\beta}\right)$ be a vecor space over the (real or complex) field $\mathbf{K}$ and a complete $\beta$-normed sapce over $\mathbf{K}$, respectively with $0<\beta \leq 1$. Let $k \geq 1$ be a given integer. Let $f: G \longrightarrow F$ be a mapping for which there exists a function $\varphi: G \times G \rightarrow[0, \infty)$ such that

$$
\left\|D_{k} f(x, y)\right\|_{\beta} \leq \varphi(x, y), \quad \forall x, y \in G .
$$

We suppose also that there exists a constant $L, 0<L<1$ such that

$$
\varphi(x, x) \leq \frac{1}{(2 k)^{\beta}} L \varphi(2 k x, 2 k x), \quad \forall x \in G
$$

and

$$
\lim _{n \rightarrow \infty}(2 k)^{n \beta} \varphi\left(\frac{x}{2^{n} k^{n}}, \frac{y}{2^{n} k^{n}}\right)=0, \quad \forall x, y \in G
$$

Then there exists a unique additive mapping $f^{*}: G \rightarrow F$ such that

$$
\left\|f(x)-f^{*}(x)\right\|_{\beta} \leq \frac{1}{2^{\beta}} \frac{L}{1-L} \varphi(x, x)
$$

for all $x \in G$.

Proof. As in the proof of Theorem 3.2, we consider $\mathbf{X}$ the set of functions from $G$ to $F$. We equipp $\mathbf{X}$ with the generalized distance $d_{\varphi}$. We know that the generalized metric space $\left(\mathbf{X}, d_{\varphi}\right)$ is complete.

We define an operator $\Lambda: \mathbf{X} \rightarrow \mathbf{X}$ by

$$
(\Lambda g)(x)=2 k g\left(\frac{x}{2 k}\right), \quad \forall x \in G .
$$

By using the same argument as in the proof of Theorem 3.2, one can prove that $\Lambda$ is a strictly contractive operator. Precisely, we have

$$
d_{\varphi}(\Lambda g, \Lambda h) \leq L d_{\varphi}(g, h), \quad \forall g, h \in \mathbf{X} .
$$

Moreover, one can prove

$$
d_{\varphi}(\Lambda f, f) \leq \frac{1}{2^{\beta}} L<\infty .
$$

instead of (3.7).

According to (iii) of Theorem 2.1, there exists a unique function $f^{*}$ : $G \rightarrow F$ in the set

$$
\mathbf{X}(f, 0):=\left\{g \in \mathbf{X}: d_{\varphi}(\Lambda f, g)<\infty\right\},
$$


which is a fixed point of $\Lambda$, such that

$$
\lim _{n \rightarrow \infty}\left(\Lambda^{n} f\right)(x)=\lim _{n \rightarrow \infty} 2^{n} k^{n} f\left(\frac{x}{2^{n} k^{n}}\right)=f^{*}(x), \quad \forall x \in G .
$$

Since the integer $n$ of Theorem 2.1 is 0 and $f \in \mathbf{X}(f, 0)$, using Theorem 2.1 (iv) and (3.15), we get

$$
d_{\varphi}\left(f, f^{*}\right) \leq \frac{1}{1-L} d_{\varphi}(\Lambda f, f) \leq \frac{1}{2^{\beta}} \frac{L}{1-L},
$$

which implies that the inequality (3.14) is true.

In order to prove that $f^{*}$ is additive, we proceed as in the last proof of Theorem 3.2 by replacing $x$ and $y$ in (3.11) by $\frac{x}{2^{n} k^{n}}$ and $\frac{y}{2^{n} k^{n}}$, respectively. We get

$$
\left\|\frac{1}{k} f\left(k\left(\frac{x+y}{2^{n} k^{n}}\right)\right)-f\left(\frac{x}{2^{n} k^{n}}\right)-f\left(\frac{y}{2^{n} k^{n}}\right)\right\|_{\beta} \leq \varphi\left(\frac{x}{2^{n} k^{n}}, \frac{y}{2^{n} k^{n}}\right),
$$

which gives after multiplying by $(2 k)^{n \beta}$ the following inequality

$$
\left\|\frac{1}{k} \Lambda^{n}(f)(k(x+y))-\Lambda^{n}(f)(x)-\Lambda^{n}(f)(y)\right\|_{\beta} \leq(2 k)^{n \beta} \varphi\left(\frac{x}{2^{n} k^{n}}, \frac{y}{2^{n} k^{n}}\right),
$$

for all $x, y \in G$.

According to (3.13) and (3.16), by letting $n$ tend to infinity in (3.17), it follows that $f^{*}(k(x+y))=k f^{*}(x)+k f^{*}(y)$, for all $x, y \in G$. Thus $f^{*}$ satisfies the functional equation (3.2) and so, by Lemma 3.1, $f^{*}$ is additive. As in the proof of Theorem 3.2, we prove that $f^{*}$ is unique. This ends the proof. $\square$ The results obtained in Theorem 3.3 complete those obtained in Theorem 3.2.

\section{Applications}

The following result concerns the Ulam-Hyers-Rassias stability of the functional equation (3.2).

Corollary 4.1. Let $(G,\|\cdot\|)$ and $\left(F,\|\cdot\|_{\beta}\right)$ be a normed vecor space over the (real or complex) field $\mathbf{K}$ and a complete $\beta$-normed space over $\mathbf{K}$, respectively, with $0<\beta \leq 1$. Let $k \geq 1$ be a given integer. Let $p$ be a real number such that $0 \leq p<\beta$. If a function $f: G \longrightarrow F$ satisfies

$$
\left\|\frac{f(k(x+y))}{k}-f(x)-f(y)\right\|_{\beta} \leq \theta\left(\|x\|^{p}+\|y\|^{p}\right), \quad \forall x, y \in G,
$$


for some given number $\theta>0$.

Then there exists a unique additive mapping $f^{*}: G \rightarrow F$ such that

$$
\left\|f(x)-f^{*}(x)\right\|_{\beta} \leq \frac{2 \theta k^{\beta}}{(2 k)^{\beta}-(2 k)^{p}}\|x\|^{p}
$$

for all $x \in G$.

Proof. We put $L:=(2 k)^{p-\beta}$. By assumption, we have $0<L<1$. We define $\varphi(x, y):=\theta\left(\|x\|^{p}+\|y\|^{p}\right)$, for all $x, y \in G$.

For every $x \in G$, we have

$$
\varphi(2 k x, 2 k x)=2(2 k)^{p} \theta\|x\|^{p}=(2 k)^{p} \varphi(x, x)=(2 k)^{\beta} L \varphi(x, x)
$$

for all $x \in G$.

In addition, we have

$$
\frac{\varphi\left(2^{n} k^{n} x, 2^{n} k^{n} y\right)}{(2 k)^{n \beta}}=L^{n} \theta\left(\|x\|^{p}+\|y\|^{p}\right) \longrightarrow 0, \text { as } n \longrightarrow \infty
$$

for any $x, y \in G$. Thus all the conditions of Theorem 3.2 are satisfied. It follows from Thorem 3.2, that there exists a unique additive function $f^{*}: G \rightarrow F$ such that the inequality (4.2) holds for all $x \in G$.

The next result concerns the case $p=0$. It is an immediate consequence of Corollary 4.1.

Corollary 4.2. Let $(G,\|\|$.$) and \left(F,\|\cdot\|_{\beta}\right)$ be a normed vecor space over the (real or complex) field $\mathbf{K}$ and a complete $\beta$-normed space over $\mathbf{K}$, respectively, with $0<\beta \leq 1$. Let $k \geq 1$ be a given integer. If a function $f: G \longrightarrow F$ satisfies

$$
\left\|\frac{f(k(x+y))}{k}-f(x)-f(y)\right\|_{\beta} \leq \delta, \quad \forall x, y \in G,
$$

for some given number $\delta>0$.

Then there exists a unique additive mapping $f^{*}: G \rightarrow F$ such that

$$
\left\|f(x)-f^{*}(x)\right\|_{\beta} \leq \frac{k^{\beta}}{(2 k)^{\beta}-1} \delta
$$

for all $x \in G$. 
Corollary 4.2 says that the functional equation (3.2) is stable in the sense of Ulam-Hyers.

In the last result, we deal with the case $p>\beta$ to complete the study of Ulam-Hyers-Rassias stability for the functional equation (3.2).

Corollary 4.3. Let $(G,\|\|$.$) and \left(F,\|.\|_{\beta}\right)$ be a normed vecor space over the (real or complex) field $\mathbf{K}$ and a complete $\beta$-normed space over $\mathbf{K}$, respectively, with $0<\beta \leq 1$. Let $k \geq 1$ be a given integer. Let $p$ be a real number such that $p>\beta$. If a function $f: G \longrightarrow F$ satisfies

$$
\left\|\frac{f(k(x+y))}{k}-f(x)-f(y)\right\|_{\beta} \leq \theta\left(\|x\|^{p}+\|y\|^{p}\right), \quad \forall x, y \in G,
$$

for some given number $\theta>0$.

Then there exists a unique additive mapping $f^{*}: G \rightarrow F$ such that

$$
\left\|f(x)-f^{*}(x)\right\|_{\beta} \leq \frac{2 \theta k^{\beta}}{(2 k)^{p}-(2 k)^{\beta}}\|x\|^{p}
$$

for all $x \in G$.

Proof. We put $L:=(2 k)^{\beta-p}$. By assumption, we have $0<L<1$. We define $\varphi(x, y):=\theta\left(\|x\|^{p}+\|y\|^{p}\right)$, for all $x, y \in G$.

For every $x \in G$, we have

$$
\varphi(x, x)=\frac{1}{(2 k)^{p}} \varphi(2 k x, 2 k x)=\frac{1}{(2 k)^{\beta}} L \varphi(2 k x, 2 k x)
$$

for all $x \in G$.

In addition, we have

$$
(2 k)^{n \beta} \varphi\left(\frac{x}{2^{n} k^{n}}, \frac{y}{2^{n} k^{n}}\right)=L^{n} \theta\left(\|x\|^{p}+\|y\|^{p}\right) \longrightarrow 0, \text { as } n \longrightarrow \infty
$$

for any $x, y \in G$. Thus all the conditions of Theorem 3.3 are satisfied. It follows from Thorem 3.3, that there exists a unique additive function $f^{*}: G \rightarrow F$ such that the inequality (4.6) holds for all $x \in G$.

Acknowledgement. The author thanks very much the anonymous referee for his (or her) helpful comments. 


\section{References}

[1] 1. M. Akkouchi, Stability of certain functional equations via a fixed point of Ćirić, Filomat, 25(2), pp. 121-127, (2011).

[2] T. Aoki, On the stability of the linear transformation in Banach spaces, J. Math. Soc. Japan, 2, pp. 64-66, (1950).

[3] J. A. Baker, The stability of certain functional equations, Proc. Amer. Math. Soc., 112 (3), pp. 729-732, (1991).

[4] D. G. Bourgin, Approximately isometric and multiplicative transformations on continuous function rings, Duke Math. J., 16, pp. 385-397, (1949).

[5] L. Cădariu and V. Radu, Fixed points and the stability of quadratic functional equations, Analele Universitatii de Vest din Timisoara, 41, pp. $25-48,(2003)$.

[6] L. Cădariu and V. Radu, Fixed points and the stability of Jensen's functional equation, J. Inequal. Pure Appl. Math., 4 (2003), Art. ID 4.

[7] L. Cădariu and V. Radu, On the stability of the Cauchy functional equation: a fixed point approach, Grazer Mathematische Berichte, 346, pp. 43-52, (2004).

[8] L. Cădariu and V. Radu, The fixed points method for the stability of some functional equations, Carpathian Journal of Mathematics, 23, pp. 63-72, (2007).

[9] P. W. Cholewa, Remarks on the stability of functional equations, Aequationes Math., 27 (1984) 76-86. MR0758860 (86d:39016).

[10] S. Czerwik, Stability of functional equations of Ulam-Hyers-Rassias type, Hadronic Press, (2003).

[11] J. B. Diaz and B. Margolis, A fixed point theorem of the alternative, for contractions on a generalized complete metric space, Bull. Amer. Math. Soc., 74, pp. 305-309, (1968). 
[12] [3] G. L. Forti, Hyers-Ulam stability of functional equations in several variables, Aequationes Math., 50, pp. 143-190, (1995).

[13] Z. Gajda, On stability of additive mappings, Internat. J. Math. Math. Sci., 14, pp. 431-434, (1991).

[14] D. H. Hyers, On the stability of the linear functional equation, Proc. Natl. Acad. Sci., 27, pp. 222-224, (1941).

[15] D. H. Hyers, G. Isac and Th. M. Rassias, Stability of functional Equations in Several Variables, Birkhauser, Boston, Basel, Berlin, (1998).

[16] G. Isac and Th. M. Rassias, On the Hyers-Ulam stability of -additive mappings, J. Approx. Theory, 72, pp. 131-137, (1993).

[17] S-M Jung, T-S Kim and K-S Lee, A fixed point approach to the stability of quadratic functional equation, Bull. Korean Math. Soc., 43(3), pp. 531-541, (2006).

[18] [14] Zs. Páles, Generalized stability of the Cauchy functional equation, Aequationes Math., 56(3), pp. 222-232, (1998).

[19] C. Park and J. M. Rassias, Stability of the Jensen-type functional equation in $C^{*}$-algebras: a fixed point approach, Abstract and Applied Analysis Volume 2009, Article ID 360432, 17 pages.

[20] V. Radu, The fixed point alternative and the stability of functional equations, Fixed Point Theory, 4, pp. 91-96, (2003).

[21] Th. M. Rassias, On the stability of the linear mapping in Banach spaces, Proc. Amer. Math. Soc., 72, pp. 297-300, (1978).

[22] J. M. Rassias, Solution of a problem of Ulam, J. Approx. Theory, 57, No. 3, pp. 268-273, (1989).

[23] Th. M. Rassias, On the stability of functional equations and a problem of Ulam, Acta Math. Appl., 62 (2000), pp. 23-130. MR1778016 (2001j:39042).

[24] Th. M. Rassias, On the stability of functional equations in Banach spaces, J. Math. Anal. Appl., 251 (2000), pp. 264-284. MR1790409 (2003b:39036).

[25] Th. M. Rassias, The problem of S.M.Ulam for approximately multiplicative mappings, J. Math. Anal. Appl., 246 (2), pp. 352-378, (2000). 
[26] I. A. Rus, Principles and Applications of Fixed Point Theory, Ed. Dacia, Cluj-Napoca, (1979) (in Romanian).

[27] [22] L. Székelyhidi, On a stability theorem, C. R. Math. Rep. Acad. Sci. Canada, 3(5), pp. 253-255, (1981).

[28] [23] L. Székelyhidi, The stability of linear functional equations, C. R. Math. Rep. Acad. Sci. Canada, 3(2), pp. 63-67, (1981).

[29] [24] L. Székelyhidi, Ulam's problem, Hyers's solution and to where they led, in Functional Equations and Inequalities, Th. M. Rassias (Ed.), Vol. 518 of Mathematics and Its Applications, Kluwer Acad. Publ., Dordrecht, pp. 259-285, (2000).

[30] S. M. Ulam, Problems in Modern Mathematics, Chapter VI, science ed. Wiley, New York, (1940).

\section{Mohamed Akkouchi}

Department of Mathematics, Cadi Ayyad University,

Faculty of Sciences-Semlalia, Av. Prince my Abdellah, B.P. 2390.

Marrakech

MAROC

e-mail : akkouchimo@yahoo.fr 